\title{
Asymptotics for the distributions of subtableaux in Young and up-down tableaux
}

\author{
David J. Grabiner \\ 7318 Eden Brook Dr. \#123 \\ Columbia, MD 21046 \\ grabiner@alumni.princeton.edu
}

Submitted: Mar 21, 2005; Accepted: Apr 2, 2006; Published: Apr 11, 2006

Mathematics Subject Classifications: primary 05E10, secondary 60G50

\begin{abstract}
Let $\mu$ be a partition of $k$, and $T$ a standard Young tableau of shape $\mu$. McKay, Morse, and Wilf show that the probability a randomly chosen Young tableau of $N$ cells contains $T$ as a subtableau is asymptotic to $f^{\mu} / k$ ! as $N$ goes to infinity, where $f^{\mu}$ is the number of all tableaux of shape $\mu$. We use a random-walk argument to show that the analogous asymptotic probability for randomly chosen Young tableaux with at most $n$ rows is proportional to $\prod_{1 \leq i<j \leq n}\left(\left(\mu_{i}-i\right)-\left(\mu_{j}-j\right)\right)$; as $n$ goes to infinity, the probabilities approach $f^{\mu} / k$ ! as expected. We have a similar formula for up-down tableaux; the probability approaches $f^{\mu} / k$ ! if $\mu$ has $k$ cells and thus the up-down tableau is actually a standard tableau, and approaches 0 if $\mu$ has fewer than $k$ cells.
\end{abstract}

\section{Introduction}

Let $\mu$ be a partition of $k$, and $T$ a standard Young tableau of shape $\mu$. We say that $T$ is a subtableau of a larger Young tableau $Y$ if the entries 1 through $k$ of $Y$ form the tableau $T$. Let $P(N, T)$ be the probability that a randomly chosen standard Young tableau of $N$ cells contains $T$ as a subtableau.

McKay, Morse, and Wilf [12] show that

$$
\lim _{N \rightarrow \infty} P(N, T)=\frac{f^{\mu}}{k !}
$$

where $f^{\mu}$ is the number of standard Young tableaux of shape $\mu$. They apply this result to problems such as the asymptotic distribution of entries in random large Young tableaux. Stanley [14] gives an exact formula for $P(N, T)$, and related asymptotics for the number of skew tableaux of a given shape. Jaggard [9] gives another proof. 
A Young tableau can be viewed as a walk in the region $x_{1} \geq x_{2} \geq \cdots \geq x_{n}$; the enumeration of walks to $\mu \in \mathbb{Z}^{n}$ is the classical $n$-candidate ballot problem. We use the random-walk view of Young tableaux, a local central limit theorem due to Kuperberg [10] which relates asymptotics for random walks and Brownian motion, and asymptotics for the Brownian motion from [6], to find a formula analogous to (1) when the number of rows of the $N$-cell tableau is restricted to $n$. The asymptotic probability is proportional to

$$
\prod_{1 \leq i<j \leq n}\left(\left(\mu_{i}-i\right)-\left(\mu_{j}-j\right)\right)
$$

where $\mu_{i}=0$ if $\mu$ has fewer than $i$ rows. As $n$ goes to infinity, we show that this approaches the probability $f^{\mu} / k$ ! in $(1)$.

We can apply (2) to compute the distributions of the entries of random large Young tableaux with at most $n$ rows. For example, the probability that the entry 2 is in the top row of a random large tableau with at most $n$ rows goes to $(n+1) / 2 n$, which approaches the expected $1 / 2$ as $n$ goes to infinity.

We have similar results for other random walks in the classical Weyl chambers. The most important case is up-down tableaux, which are the analogue of Young tableaux in the representation theory of the symplectic group [1, 7, 15]. An up-down tableau [15] of size $k$ on $n$ rows is a sequence of $k$ partition diagrams each with at most $n$ rows, in which the first has only one cell, and each subsequent tableau is obtained by either adding or deleting one cell. Just as the number of standard tableaux of shape $\mu$ of size $k$ is the multiplicity of the representation with highest weight $\mu$ in the $k$ th tensor power of the defining representation of $G L_{n}$, the number of up-down tableaux of shape $\mu$ of size $k$ on at most $n$ rows is the multiplicity of the representation with highest weight $\mu$ in the $k$ th tensor power of the defining representation of $S p_{2 n}[1,7]$.

The result for up-down tableaux is not quite the same as for standard tableaux. In a random up-down tableau of size $N$ with at most $n$ rows, the probability that the tableau at step $k$ is actually a standard Young tableau (with only upward steps, and thus $k$ cells) goes to 1 as the number of rows $n$ goes to infinity. The limiting distribution among these tableaux is the same as in random standard tableaux of size $N$; thus the limit still approaches $f^{\mu} / k$ !, where $\mu$ is restricted to those shapes which are actually partitions of $k$.

\section{Random walks in Weyl chambers}

We will give the necessary definitions and properties of Weyl groups from [8], and of random walks in Weyl chambers from [7].

A Coxeter group $W$ is a discrete group generated by reflections in $\mathbb{R}^{n}$. It is determined by the root system $\Phi$, a set of vectors orthogonal to the hyperplanes of reflection, or by the positive roots $\Phi^{+}$, choosing only one root for each hyperplane of reflection so that all the positive roots are on the same side of a given hyperplane. If $W$ stabilizes a lattice, it is called a Weyl group, and the hyperplanes of reflection partition space into Weyl chambers.

For example, the roots $x_{i}-x_{j}$ in $\mathbb{R}^{n}$ give reflections in the hyperplanes $x_{i}=x_{j}$, and 
these generate the symmetric group $S_{n}$ (called $A_{n-1}$ as a Weyl group). The principal Weyl chamber is $x_{1}>x_{2}>\cdots>x_{n}$.

We consider the enumeration of walks which stay inside the Weyl chamber, or equivalently the probability distribution for a random walk which is killed when it hits a wall of the Weyl chamber. Such a random walk is reflectable [7] if the step set $S$ is symmetric under the Weyl group $W$, and the steps and starting point are such that a step which starts in the interior of the Weyl chamber cannot cross a wall. For example, on the lattice $\mathbb{Z}^{n}$, the walk with Weyl group $S_{n}$ and steps $e_{i}$ in the positive coordinate directions is reflectable; a step starting at a point with $x_{i}>x_{j}$ can go to a point with $x_{i}=x_{j}$ but not a point with $x_{i}<x_{j}$.

The ballot problem can be converted to a reflectable walk problem by translating the walk to start at $\eta=(-1,-2, \ldots,-n)$ and end at $\lambda=\mu+\eta$; the condition $x_{i} \geq x_{i+1}$ before the translation becomes $x_{i} \geq x_{i+1}+1$ or equivalently $x_{i}>x_{i+1}$.

Definitions. Let $b_{\eta \lambda, k}$ be the number of walks of length $k$ from $\eta$ to $\lambda$ with a step set $S$ which stay in the Weyl chamber. Let $c_{\gamma, k}$ be the number of walks of length $k$ from the origin to $\gamma$ (or equivalently with any start and end with difference $\gamma$ ) with the same step set $S$, but unconstrained by a chamber. Similarly, for Brownian motion, let $b_{t}(\eta, \lambda)$ be the density function for $n$-dimensional Brownian motion started at $\eta$ to be at $\lambda$ at time $t$, staying within the Weyl chamber through that time. Let $c_{t}(\gamma)$ be the density function for $n$-dimensional Brownian motion to go from the origin to $\gamma$ at time $t$, unconstrained by a chamber.

For reflectable walks, Gessel and Zeilberger [5] and independently Biane [2] related the number of constrained walks to a signed sum of unconstrained walks of the same length. The formula is

$$
b_{\eta \lambda, k}=\sum_{w \in W} \operatorname{sgn}(w) c_{\lambda-w(\eta), k} .
$$

The proof is analogous to the reflection argument for the Catalan numbers [5]. Every walk from any $w(\eta)$ to $\lambda$ which does touch at least one wall has some last step $j$ at which it touches a wall; let the wall be the hyperplane perpendicular to $\alpha_{i}$, choosing the largest $i$ if there are several choices [13]. Reflect all steps of the walk up to step $j$ across that hyperplane; the resulting walk is a walk from $w_{\alpha_{i}} w(\eta)$ to $\lambda$ which also touches wall $i$ at step $j$. This clearly gives a pairing of walks, and since $w_{\alpha_{i}}$ has sign -1 , these two walks cancel out in (3). The only walks which do not cancel in these pairs are the walks which stay within the Weyl chamber, and since $w(\eta)$ is inside the Weyl chamber only if $w$ is the identity, this is the desired number of walks.

An analogous result with an analogous proof holds for Brownian motion, which is always reflectable because it is continuous and symmetric under all reflections. The formula is $[6]$

$$
b_{t}(\eta, \lambda)=\sum_{w \in W} \operatorname{sgn}(w) c_{t}(\lambda-w(\eta))
$$

(This theorem is stated in [6] with $c_{t}(w(\lambda)-\eta)$ instead of $c_{t}(\lambda-w(\eta))$, reflecting the Brownian motion after the first time it hits a wall rather than before the last time. The 
two forms are equivalent since Brownian motion is symmetric under the Weyl group, and we will need to use the theorem in the form (4) later.)

The step sets which give reflectable walks are enumerated in [7]; they turn out to be precisely the Weyl group images of the minuscule weights [3], those weights with dot-product 0 or \pm 1 with every root. The reflectable walks include the weights which are minuscule for only one of $B_{n}$ and $C_{n}$, which are the same as Weyl groups but have different root systems. In the Bourbaki numbering [3], the allowed step sets are the Weyl group images of the following $\check{\omega}_{i}$, the duals of the fundamental roots.

$$
\begin{array}{rcl}
A_{n}: & \check{\omega}_{1}, \ldots, \check{\omega}_{n} . & \text { All compatible. } \\
B_{n}, C_{n}: & \check{\omega}_{1}, \check{\omega}_{n} . & \text { Not compatible. } \\
D_{n}: & \check{\omega}_{1}, \check{\omega}_{n-1}, \check{\omega}_{n} . & \text { All compatible. } \\
E_{6}: & \check{\omega}_{1}, \check{\omega}_{6} . & \text { Compatible. } \\
E_{7}: & \check{\omega}_{7} . & \\
E_{8}, F_{4}, G_{2}: & \text { None. } &
\end{array}
$$

Any union of compatible step sets also gives a reflectable random walk.

The classical Weyl groups are $A_{n-1}=S_{n}, B_{n}=C_{n}$, and $D_{n}$. The Weyl group $A_{n-1}$ has roots $x_{i}-x_{j}$ and principal Weyl chamber $x_{1}>x_{2}>\cdots>x_{n}$. The Weyl group $B_{n}$ has roots $x_{i} \pm x_{j}$ and $x_{i}$. It contains all permutations with any number of sign changes, and has principal Weyl chamber $x_{1}>x_{2}>\cdots>x_{n}>0$. The Weyl group $D_{n}$ has roots $x_{i} \pm x_{j}$. It contains all permutations with an even number of sign changes, and has principal Weyl chamber $x_{1}>x_{2}>\cdots>x_{n}, x_{n-1}>-x_{n}$.

The group $A_{n-1}=S_{n}$ acts on the hyperplane $H$ given by $\sum x_{i}=0$, a subspace of $\mathbb{R}^{n}$. Thus a random walk on $\mathbb{R}^{n}$ is reflectable if the steps project onto reflectable steps on $H$, and the step set is symmetric under $S_{n}$. In particular, the steps $e_{i}$ give a reflectable random walk; this is the most important case because it is the walk we use for Young tableaux. The step $e_{1}$ projects to the fundamental weight $\check{\omega}_{1}=((n-1) / n,-1 / n, \ldots,-1 / n)$, and the other steps project to its $S_{n}$-images. This step set is not reflectable for $B_{n}$ or $D_{n}$ because it is not symmetric under those Weyl groups.

The walk we use for up-down tableaux has step set $\pm e_{i}$ on $\mathbb{Z}^{n}$, which gives a reflectable random walk for all three groups. The step $e_{1}$ is the fundamental weight $\check{\omega}_{1}$ for $B_{n}$ and $D_{n}$, and $e_{1}$ and $-e_{1}$ project to the fundamental weights $\check{\omega}_{1}$ and $\check{\omega}_{n-1}$ for $A_{n-1}$.

\section{Asymptotics for random walks and Brownian mo- tion}

We continue throughout the paper to let $\mu$ be a partition of $k$. Let $\beta=(-1,-2, \ldots,-n)$, so that the number of Young tableaux of shape $\mu$ is the number of walks in the region $x_{1}>x_{2}>\cdots>x_{n}$ from $\beta$ to $\beta+\mu$. A Young tableau with $N$ cells and at most $n$ rows corresponds to a walk starting from $\beta$ of $N$ steps, and the number of Young tableaux with $N$ cells which contain a specific subtableau of shape $\mu$ is thus the number of walks 
starting at $\beta+\mu$ which stay in the Weyl chamber $x_{1}>x_{2}>\cdots>x_{n}$ for $N-k$ more steps.

We now view this as a problem in random walks, taking each step randomly in one of the coordinate directions. The probability that a walk of length $N$ will reach a tableau of shape $\mu$ in $k$ steps is $f^{\mu} / n^{k}$, since each of the $f^{\mu}$ tableaux corresponds to one walk. Applying Bayes' Theorem,

$$
\begin{aligned}
P(n, N, T) & =P(\mu \text { at step } k \mid \text { survive to step } N) / f^{\mu} \\
& =\frac{P(\text { survive to step } N \mid \mu \text { at step } k) P(\mu \text { at step } k) / f^{\mu}}{P(\text { survive to step } N)} \\
& =\frac{P(\text { survive to step } N \mid \mu \text { at step } k)}{n^{k} P(\text { survive to step } N)} .
\end{aligned}
$$

The denominator of the last fraction is independent of $\mu$. Thus the probability $P(n, N, T)$ for tableaux of shape $\mu$ is proportional to the probability that a random walk which reaches $\mu$ at step $k$ will survive to step $N$.

It is more natural to compute the asymptotic for the probability that a random walk started at $\mu$ will survive for $N$ more steps, since this is a more natural problem and the notation will be simpler; we will replace $N$ in the asymptotic by $N-k$ when we apply (5) to our specific problem for Young tableaux. We can compute asymptotics for these probabilities up to a constant factor; we can then eliminate the constant because $\sum_{T} P(n, N, T)=1$ by definition. We will compute the asymptotics for this case, and by an analogous process for the other classical Weyl groups.

Theorem 1 For any reflectable random walk in any of the classical Weyl chambers, where the Weyl group has $m$ positive roots, the asymptotic probability that a walk starting at $\eta$ will stay in the chamber for some large number $N$ of steps is

$$
C N^{-m / 2} \prod_{\alpha \in \Phi^{+}} \alpha(\eta)
$$

for a constant $C$ depending on the walk and the chamber. This is the same as the asymptotic for Brownian motion (appropriately normalized so that the random walk and the Brownian motion have the same variance) started at $\eta$ in the same chamber to remain in the chamber up to time $N$.

We conjecture that this theorem also holds for reflectable walks for the exceptional groups $E_{6}$ and $E_{7}$.

We will start with the asymptotics for the corresponding problem in Brownian motion, the probability that a Brownian motion started at $\eta$ will stay within the Weyl chamber up to a large time $t$. These asymptotics are given by the following lemmas from [6].

Lemma 1 For Brownian motion in any of the classical Weyl chambers in $\mathbb{R}^{n}$, where the Weyl group has $m$ positive roots, we have the following asymptotic for the density $b_{t}(\eta, \lambda)$ 
for the motion started at $\eta$ to stay inside the chamber up to time $t$, with different constants $C$ for the different chambers:

$$
b_{t}(\eta, \lambda) \approx C t^{-m-n / 2} \prod_{\alpha \in \Phi^{+}} \alpha(\lambda) \alpha(\eta) \exp \left(\frac{-|\lambda|^{2}-|\eta|^{2}}{2 t}\right) .
$$

For $|\lambda|$ of order $O\left(t^{\epsilon+1 / 2}\right)$, this asymptotic is valid to a factor of $1+O\left(t^{\epsilon-1 / 2}\right)$.

We omit the details of the computation because it is a separate, very long computation for each classical Weyl group. The computations of the asymptotics in [6] do not generalize naturally to the exceptional groups, which is part of the reason we can only conjecture that Theorem 1 holds for $E_{6}$ and $E_{7}$; if the asymptotic has the correct form and Lemma 3 below also holds, the rest of the proof goes through unchanged.

Lemma 2 For Brownian motion in any of the classical Weyl chambers, the probability that the motion started at $\eta$ will survive to time $t$ is asymptotic to

$$
C t^{-m / 2} \prod_{\alpha \in \Phi^{+}} \alpha(\eta)
$$

again with a constant $C$ (different from the constant in Lemma 1) which depends on the chamber.

This lemma is proved in [6] by integrating the asymptotic from Lemma 1 over the Weyl chamber. Since we need the same arguments from the proof of Lemma 2 in our proof of Theorem 1, we will include the full proof of Lemma 2 there.

As examples of these lemmas, for the symmetric group, which has $m=n(n-1) / 2$ roots, the asymptotic density is

$$
C t^{-n^{2} / 2} \prod_{i<j}\left(\lambda_{i}-\lambda_{j}\right)\left(\eta_{i}-\eta_{j}\right)
$$

and the asymptotic probability that the motion stays inside the chamber is

$$
C t^{-n(n-1) / 4} \prod_{i<j}\left(\eta_{i}-\eta_{j}\right)
$$

We now use a local central limit theorem of Kuperberg [10] to relate these asymptotics to asymptotics for the random walk. We expect Brownian motion to approximate a random walk for large time. That is, if the steps in the random walk are all in $\vec{z}+L$ for some constant $\vec{z}$ and the thinnest possible lattice $L$, then after $N$ steps, the random walk must be in $N \vec{z}+L$. Let det $L$ be the determinant of a positive basis for $L$, which is the volume of the quotient space $\mathbb{R}^{n} / L$. We can thus tile $\mathbb{R}^{n}$ with regions of volume $\operatorname{det} L$ such that each region contains one point in $N \vec{z}+L$ for each value of $N$, and thus only one possible point for the random walk to reach at step $N$. As $N$ goes to infinity, we expect that the probability that the random walk is in this region at step $N$ and the probability 
that the corresponding Brownian motion is in this region at time $N$ to converge. The local central limit theorem gives the rate of convergence, both for single random walks and for signed sums like those in (3) and (4).

A finite difference operator $D$ is a linear operator on functions from $\mathbb{R}^{n}$ to $\mathbb{R}$ defined by a finite sum

$$
D f(\vec{v})=\sum_{i} a_{i} f\left(\vec{v}+\vec{v}_{i}\right)
$$

for some constants $a_{i}$ and vectors $\vec{v}_{i}$. The degree of $D$ is the minimum degree of a polynomial $p$ such that $D p \neq 0$.

We have the following slightly stronger statement of a theorem [10, Theorem 4] which generalizes a proof in [11, Theorem 1.2.1].

Theorem 2 Let $X$ be a bounded, mean 0 random variable taking values in $\vec{z}+L$ for some lattice $L \in \mathbb{R}^{n}$, with $L$ the thinnest possible lattice for $X$. Let $\kappa$ be the covariance form of $X$. Let $Y_{N}$ be the sum of $N$ independent copies of $X$. For any vector $\vec{v}$, let $p(\vec{v})$ be the probability for the random walk, and let $q(\vec{v})$ the density for the corresponding Brownian motion multiplied by the factor $\operatorname{det} L$ (since $1 / \operatorname{det} L$ is the density of the support of $Y_{N}$ ); that is,

$$
p(\vec{v})=P\left(Y_{N}=\vec{v}\right), q(\vec{v})=\frac{\operatorname{det} L}{(2 \pi)^{n / 2} \sqrt{\operatorname{det} \kappa}} e^{-\kappa^{-1}(\vec{v}, \vec{v}) / 2 N} .
$$

Then for any finite difference operator $D$ of degree $d$, and any integer $b \geq 0$,

$$
D(p-q)(\vec{v})|\vec{v}|^{b}=O\left(N^{(-n+b-d-2) / 2}\right),
$$

uniformly for all $\vec{v} \in N \vec{z}+L$.

The statement of this theorem in [10] has a weaker bound,

$$
\lim _{N \rightarrow \infty} N^{(n-b+d) / 2}|\vec{v}|^{b} D(p-q)(\vec{v})=0 .
$$

Our statement in Theorem 2 is stronger because it gives a rate of convergence of $O(1 / N)$ in (13). However, the original theorem statement in $[11,1.10-1.15]$ has the stronger bound in the cases it covers (the random walk on $\mathbb{Z}^{n}$ with steps in the positive and negative coordinate directions, $b=0$ or $b=2$, and $d=0, d=1$, or a restricted case with $d=2$ ). And as [10] notes, the proof in [11] carries over in its full generality for even $b$, and the theorem follows for odd $b$ by taking the geometric mean of the formulas for $b-1$ and $b+1$. It thus establishes the stronger bound.

To apply Theorem 2 in our cases, let $b=0$, and let

$$
D f(\lambda)=\sum_{w \in W} \operatorname{sgn}(w) f(\lambda-w(\eta)) .
$$

This gives the sums we have in (3) and (4).

Lemma 3 The degree of the difference operator $D$ is the number $m$ of positive roots if the Weyl group $W$ is any of the classical groups. 
As with Lemma 1, we conjecture that this lemma holds for other Weyl groups, but we need to find the degree of $D$ explicitly for each group and our arguments do not generalize naturally to the exceptional groups.

We will show that $D f=0$ if $f$ is a monomial $f(\lambda)=\prod_{i} \lambda_{i}^{d_{i}}$ of degree less than $m$. To show that the degree of $D$ is actually $m$, we let $f(\lambda)=\prod_{\alpha \in \Phi^{+}} \alpha(\lambda)$; this polynomial of degree $m$ has $D f(0)=|W| \prod_{\alpha \in \Phi^{+}}(\alpha(-\eta)) \neq 0$.

For the group $A_{n-1}$, the Weyl group is the symmetric group, and (14) becomes

$$
D f(\lambda)=\sum_{\sigma \in S_{n}} \operatorname{sgn}(\sigma) \prod_{i}\left(\lambda_{i}-\eta_{\sigma(i)}\right)^{d_{i}} .
$$

This is a determinant

$$
\operatorname{det}_{n \times n}\left|\left(\lambda_{i}-\eta_{j}\right)^{d_{i}}\right| .
$$

Expand each term by the binomial theorem to get

$$
\operatorname{det}_{n \times n}\left|\sum_{k}\left(\begin{array}{c}
d_{i} \\
k
\end{array}\right)\left(-\eta_{j}\right)^{k}\left(\lambda_{i}\right)^{d_{i}-k}\right| \text {. }
$$

Row $i$ of this matrix is a sum of terms for $d_{i}+1$ different values of $k$. We can thus write the single determinant as a sum of $\prod\left(d_{i}+1\right)$ determinants, choosing one value of $k_{i}$ as the $k$ for each row,

$$
\sum_{k_{i} \leq d_{i}} \operatorname{det}_{n \times n}\left|\left(\begin{array}{c}
d_{i} \\
k_{i}
\end{array}\right)\left(-\eta_{j}\right)^{k_{i}}\left(\lambda_{i}\right)^{d_{i}-k_{i}}\right| .
$$

If $k_{i}=k_{i^{\prime}}$, then rows $i$ and $i^{\prime}$ in the matrix are

$$
\left(\begin{array}{l}
d_{i} \\
k_{i}
\end{array}\right)\left(-\eta_{j}\right)^{k_{i}}\left(\lambda_{i}\right)^{d_{i}-k_{i}} \text { and }\left(\begin{array}{c}
d_{i^{\prime}} \\
k_{i}
\end{array}\right)\left(-\eta_{j}\right)^{k_{i}}\left(\lambda_{i^{\prime}}\right)^{d_{i^{\prime}}-k_{i}}
$$

which are constant multiples of one another because everything except $\left(-\eta_{j}\right)^{k_{i}}$ is constant across the row. Thus every determinant in (18) is zero unless the $k_{i}$ are all different, and $n$ different non-negative integers must sum to at least $n(n-1) / 2$. Since $d_{i} \geq k_{i}$, the sum of the $d_{i}$ must also be at least $n(n-1) / 2$. Thus $D f=0$ if $f$ is of degree less than $n(n-1) / 2$, which is the number $m$ of positive roots for the group $A_{n-1}$.

The argument for the other two groups is similar. For $B_{n}$, an element of the Weyl group can be written as the product of a permutation $\sigma$ and any number of sign changes, so (14) becomes

$$
D f(\lambda)=\sum_{\sigma \in S_{n}} \sum_{\epsilon_{i}= \pm 1} \operatorname{sgn}(\sigma) \prod_{i} \epsilon_{i} \prod_{i}\left(\lambda_{i}-\epsilon_{i} \eta_{\sigma(i)}\right)^{d_{i}}
$$

Combining the $\epsilon_{i}= \pm 1$ terms into a single term gives

$$
D f(\lambda)=\sum_{\sigma \in S_{n}} \operatorname{sgn}(\sigma) \prod_{i}\left[\left(\lambda_{i}-\eta_{\sigma(i)}\right)^{d_{i}}-\left(\lambda_{i}+\eta_{\sigma(i)}\right)^{d_{i}}\right]
$$


Again, this is a determinant, and we expand the terms by the binomial theorem to get

$$
\operatorname{det}_{n \times n}\left|\left(\lambda_{i}-\eta_{j}\right)^{d_{i}}-\left(\lambda_{i}+\eta_{j}\right)^{d_{i}}\right|=\operatorname{det}_{n \times n}\left|\sum_{k \text { odd }}-2\left(\begin{array}{c}
d_{i} \\
k
\end{array}\right) \eta_{j}^{k}\left(\lambda_{i}\right)^{d_{i}-k}\right| \text {. }
$$

Again, we write row $i$ as a sum of values for different $k_{i}$, and write the determinant as a sum of determinants

$$
\sum_{\text {odd } k_{i} \leq d_{i}} \operatorname{det}_{n \times n}\left|-2\left(\begin{array}{c}
d_{i} \\
k_{i}
\end{array}\right) \eta_{j}^{k_{i}}\left(\lambda_{i}\right)^{d_{i}-k_{i}}\right| .
$$

If $k_{i}=k_{i}^{\prime}$, then rows $i$ and $i^{\prime}$ are constant multiples of one another, just as in (18). Thus every determinant in (23) is zero unless the $k_{i}$ are all different, and $n$ different positive odd integers must sum to at least $n^{2}$. Since $d_{i} \geq k_{i}$, the sum of the $d_{i}$ must also be at least $n^{2}$. Thus $D f=0$ if $f$ is of degree less than $n^{2}$, which is the number $m$ of positive roots for the group $B_{n}$.

For $D_{n}$, an element of the Weyl group can be written as the product of a permutation $\sigma$ and an even number of sign changes, so (14) becomes

$$
D f(\lambda)=\sum_{\sigma \in S_{n}} \sum_{\epsilon_{i}= \pm 1} \operatorname{sgn}(\sigma)\left(\frac{1+\prod_{i} \epsilon_{i}}{2}\right) \prod_{i}\left(\lambda_{i}-\epsilon_{i} \eta_{\sigma(i)}\right)^{d_{i}} .
$$

The $\prod_{i} \epsilon_{i} / 2$ terms are the same as for $B_{n}$, so they sum to zero if $f$ is of degree less than $n^{2}$. The other terms sum to

$$
\frac{1}{2} \sum_{\sigma \in S_{n}} \operatorname{sgn}(\sigma) \prod_{i}\left[\left(\lambda_{i}-\eta_{\sigma(i)}\right)^{d_{i}}+\left(\lambda_{i}+\eta_{\sigma(i)}\right)^{d_{i}}\right] .
$$

Yet again, this is a determinant, and we expand the terms by the binomial theorem to get

$$
\frac{1}{2} \operatorname{det}_{n \times n}\left|\left(\lambda_{i}-\eta_{j}\right)^{d_{i}}+\left(\lambda_{i}+\eta_{j}\right)^{d_{i}}\right|=\operatorname{det}_{n \times n}\left|\sum_{k \text { even }}\left(\begin{array}{c}
d_{i} \\
k
\end{array}\right) \eta_{j}^{k}\left(\lambda_{i}\right)^{d_{i}-k}\right| .
$$

Again, we write row $i$ as a sum of values for different $k_{i}$, and write the determinant as a sum of determinants

$$
\sum_{\text {even } k_{i} \leq d_{i}} \operatorname{det}_{n \times n}\left|\left(\begin{array}{l}
d_{i} \\
k_{i}
\end{array}\right) \eta_{j}^{k_{i}}\left(\lambda_{i}\right)^{d_{i}-k_{i}}\right|
$$

If $k_{i}=k_{i}^{\prime}$, then rows $i$ and $i^{\prime}$ are constant multiples of one another, just as in (18). Thus every determinant in (27) is zero unless the $k_{i}$ are all different, and $n$ different non-negative even integers must sum to at least $n^{2}-n$. Since $d_{i} \geq k_{i}$, the sum of the $d_{i}$ must also be at least $n^{2}-n$. Thus $D f=0$ if $f$ is of degree less than $n^{2}-n$, which is the number $m$ of positive roots for the group $D_{n}$. This completes the proof of Lemma 3 .

We can apply Theorem 2 directly for the cases with steps $\pm e_{i}$, but for the case with steps $e_{i}$, the random variable does not have mean 0 . We project the random walk and Brownian motion onto the hyperplane $H$ given by $\sum x_{i}=0$, so that the steps are the $n$ 
vectors with one coordinate $(n-1) / n$ and all others $-1 / n$; this random walk, now in $n-1$ dimensions, has mean 0 . The starting point projects to $((1-n) / 2,(3-n) / 2, \ldots,(n-$ $3) / 2,(n-1) / 2)$. The lattice $L$ on $H$ is the lattice $A_{n-1}$ of all points in $\mathbb{Z}^{n}$ with $\sum x_{i}=0$; if $n$ is even, the walk is actually on $\left(\mathbb{Z}+\frac{1}{2}\right)^{n} \cap H$, a translation of $L$.

Since all the walls of the $A_{n-1}$ Weyl chamber are orthogonal to $H$, the random walk or Brownian motion projected onto $H$ will hit a wall with the same probability as the walk or Brownian motion on $\mathbb{R}^{n}$. We thus compute our probabilities for walks projected to $H$. Let $\vec{y}=(1 / \sqrt{n}, \cdots, 1 / \sqrt{n})$ be the unit normal vector to $H$, so that we can write $\lambda=\lambda_{H}+\lambda_{\vec{y}} \vec{y}$, where $\lambda$ is the projection of $\lambda$ onto $H$, and similarly for $\eta$. The walk on $H$ now goes from $\eta_{H}$ to $\lambda_{H}$. Note that $\alpha(\lambda)=\alpha\left(\lambda_{H}\right)$ for each root $\alpha$ since $\alpha(\vec{y})=0$. Also note that by orthogonality, $|\lambda|^{2}=\left|\lambda_{H}\right|^{2}+\left|\lambda_{\vec{y}}\right|^{2}$.

In order to apply our Brownian motion results, we need the Brownian motion to be a scaling of standard Brownian motion, and thus we need the covariance form $\kappa$ to be a multiple of the identity. We can easily show that it is a multiple of the identity for any walk with a step set symmetric under the Weyl group. We will also compute det $L$ and $\kappa$ explicitly for the most important cases. We need to know $\kappa$ in order to scale the Brownian motion appropriately; it turns out that $\operatorname{det} L$ cancels out and does not even affect the constant factor $C$ in Theorem 1 .

To see that $\kappa$ is a multiple of the identity for $A_{n-1}$, consider the basis $\vec{v}_{i}=e_{1}-e_{i+1}$, which is natural but not orthogonal. If $n=2$, there is only one dimension and thus $\kappa$ is a scalar. If $n \geq 3$, then in order to have zero covariance, we need to have $E\left(\vec{v}_{1} \cdot X\right)\left(\vec{v}_{2}^{\prime} \cdot X\right)=0$ (and similarly for other pairs), where $X$ is a random step, and $\vec{v}_{2}^{\prime}$ is $\vec{v}_{2}$ minus its orthogonal projection onto $\vec{v}_{1}$. This gives $\vec{v}_{2}^{\prime}=\frac{1}{2} e_{1}+\frac{1}{2} e_{2}-e_{3}$. Now let $\sigma \in S_{n}$ switch the first two coordinates. Then $\vec{v}_{1} \cdot X=-\vec{v}_{1} \cdot \sigma(X)$, and $\vec{v}_{2}^{\prime} \cdot X=\vec{v}_{2}^{\prime} \cdot \sigma(X)$, so $E\left(\vec{v}_{1} \cdot X\right)\left(\vec{v}_{2}^{\prime} \cdot X\right)=$ $-E\left(\vec{v}_{1} \cdot \sigma(X)\right)\left(\vec{v}_{2}^{\prime} \cdot \sigma(X)\right)$, and thus the expected value is zero. Thus $\kappa$ is diagonal, and by symmetry in the coordinates, it is a multiple of the identity.

For $B_{n}$ and $D_{n}$, it is even easier to show zero covariance, because we can use the standard basis of $\mathbb{R}^{n}$. We need $E\left(x_{1} x_{2}\right)=0$ for a random step $X$. $B_{n}$ has the root $x_{1}$, so it contains a reflection which changes the sign of $x_{1}$, and thus of $x_{1} x_{2}$. $D_{n}$ for $n \geq 3$ has roots $x_{1}+x_{3}$ and $x_{1}-x_{3}$; reflecting a step in both roots changes the sign of $x_{1}$ and $x_{3}$, so it also changes the sign of $x_{1} x_{2}$. Thus, in both cases, we pair steps with opposite values of $x_{1} x_{2}$, so $E\left(x_{1} x_{2}\right)=0$, and thus $\kappa$ is diagonal and must be a multiple of the identity. ( $D_{2}$ does not have zero covariance in $\mathbb{R}^{2}$, but it is the group $A_{1}$.)

For the walk with steps $e_{i}$ and Weyl group $A_{n-1}$, the covariance form $\kappa$ is $1 / n$ times the identity when we consider the walk on $\mathbb{R}^{n}$, and it remains $1 / n$ times the identity after projection onto $H$; the hyperplane $H$ has $n-1$ dimensions but the steps have length $\sqrt{(n-1) / n}$. To find $\operatorname{det} L$, note that a basis for $L$ is $e_{1}-e_{i+1}$, and the vector $\vec{y}$ has 
length 1 and is orthogonal to all vectors in $L$. Thus

$$
\operatorname{det} L=\operatorname{det}\left|\begin{array}{rrrrr}
1 & -1 & 0 & \cdots & 0 \\
1 & 0 & -1 & \cdots & 0 \\
\vdots & \vdots & \vdots & \ddots & \vdots \\
1 & 0 & 0 & \cdots & -1 \\
1 / \sqrt{n} & 1 / \sqrt{n} & 1 / \sqrt{n} & \cdots & 1 / \sqrt{n}
\end{array}\right| .
$$

Adding $1 / \sqrt{n}$ times every other row to the last row gives

$$
\operatorname{det} L=\operatorname{det}\left|\begin{array}{rrrrr}
1 & -1 & 0 & \cdots & 0 \\
1 & 0 & -1 & \cdots & 0 \\
\vdots & \vdots & \vdots & \ddots & \vdots \\
1 & 0 & 0 & \cdots & -1 \\
\sqrt{n} & 0 & 0 & \cdots & 0
\end{array}\right|=\sqrt{n} .
$$

For the walk with steps $\pm e_{i}$, with any of the Weyl groups, the lattice $L$ is the lattice $D_{n} \subset \mathbb{Z}^{n}$ of all integer points with $\sum x_{i}$ even (since the steps are all in $e_{1}+L$ ), so $\operatorname{det} L=2$. The covariance form $\kappa$ is $1 / n$ times the identity since each step has probability $1 / n$ of being in any of the $n$ coordinate directions.

With all of the necessary constants computed, we are now ready to prove Theorem 1 itself. Let $N$ be the number of steps for the random walk. Since the covariance form $\kappa$ is a multiple of the identity $\left(1 / n\right.$ for the cases with steps $e_{i}$ or $\left.\pm e_{i}\right)$, we write it as a scalar. Thus the exponential factor in $q(\vec{v})$ is $\exp \left(-|\vec{v}|^{2} /(2 \kappa N)\right)$, corresponding to standard $n$ dimensional Brownian motion at time $\kappa N$. In order to get the random walk and Brownian motion to have the same variance, we let the time $t$ for standard Brownian motion be $\kappa N$; equivalently, we could consider standard Brownian motion scaled by a factor of $\sqrt{\kappa}$ at time $N$.

Theorem 2 says that for sufficiently large $N$, the random-walk and Brownian-motion probabilities converge at a rate of $O\left(N^{(-m-n-2) / 2}\right)$ since $D$ is of degree $m$; that is, for a walk in $n$ dimensions with step set $S$, in the notation of (3) and (4),

$$
\left.\left|b_{\eta \lambda, N} /\right| S\right|^{N}-b_{\kappa N}(\eta, \lambda) \operatorname{det} L \mid=O\left(N^{(-m-n-2) / 2}\right),
$$

and for a walk restricted to the $(n-1)$-dimensional subspace $H$,

$$
\left.\left|b_{\eta_{H} \lambda_{H}, N} /\right| S\right|^{N}-b_{\kappa N}\left(\eta_{H}, \lambda_{H}\right) \operatorname{det} L \mid=O\left(N^{(-m-n-1) / 2}\right) .
$$

The factor of $\sqrt{\operatorname{det} \kappa}$ in Theorem 2 was a normalizing factor to make the integral of the Brownian motion over $\mathbb{R}^{n}$ equal to 1 ; it thus becomes part of the $b_{\kappa N}$ term in the formulas (30) and (31).

We will now prove Lemma 2, computing the probability that Brownian motion stays within the Weyl chamber by integrating $b_{\kappa N}(\eta, \lambda)$ over all $\lambda$ in the Weyl chamber, and then relate this Brownian motion integral to the sum for random walks. 
The probability that Brownian motion stays within the Weyl chamber for time $\kappa N$ is

$$
\int_{\text {chamber }} b_{\kappa N}(\eta, \lambda) d \lambda
$$

If the Weyl group is $A_{n-1}$, we can do the $n$-dimensional integral by first integrating along $\vec{y}$; the integrand in that direction is a normal distribution. Intuitively, this is true because we could apply the reflection argument to the Brownian motion on $H$ instead of on $\mathbb{R}^{n}$. Formally, by (4),

$$
b_{\kappa N}(\eta, \lambda)=\sum_{\sigma \in S_{n}} \operatorname{sgn}(\sigma) c_{\kappa N}(\lambda-\sigma(\eta)) .
$$

We can factor out the direction $\vec{y}$ from the standard Brownian motion density $c_{\kappa N}$, writing

$$
c_{\kappa N}(\lambda-\sigma(\eta))=c_{\kappa N}\left(\lambda_{H}-\sigma\left(\eta_{H}\right)\right) c_{\kappa N}\left(\lambda_{\vec{y}} \vec{y}-\sigma\left(\eta_{\vec{y}} \vec{y}\right)\right),
$$

where the two factors are $(n-1)$-dimensional and 1-dimensional Brownian motions. And $\vec{y}$ is invariant under permutations, so we have in fact

$$
c_{\kappa N}(\lambda-\sigma(\eta))=c_{\kappa N}\left(\lambda_{H}-\sigma\left(\eta_{H}\right)\right) c_{\kappa N}\left(\lambda_{\vec{y}}-\eta_{\vec{y}}\right) .
$$

The factor $c_{\kappa N}\left(\lambda_{\vec{y}}-\eta_{\vec{y}}\right)$ is the density of a one-dimensional Brownian motion started at $\eta_{\vec{y}}$. It thus integrates to 1 over $\mathbb{R}$, and thus we can remove the innermost integral from the multiple integral,

$$
\begin{gathered}
\int_{H \cap \text { chamber }} \int_{\mathbb{R}_{\sigma \in S_{n}}} \sum_{\operatorname{sgn}(\sigma) c_{\kappa N}\left(\lambda_{H}-\sigma\left(\eta_{H}\right)\right) c_{\kappa N}\left(\lambda_{\vec{y}}-\eta_{\vec{y}}\right) d \lambda_{\vec{y}} d \lambda_{H}=} \\
\int_{H \cap \text { chamber }} \sum_{\sigma \in S_{n}} \operatorname{sgn}(\sigma) c_{\kappa N}\left(\lambda_{H}-\sigma\left(\eta_{H}\right)\right) d \lambda_{H} .
\end{gathered}
$$

The integrand is in the correct form (4), so we may write this last integral as

$$
\int_{H \cap \text { chamber }} b_{\kappa N}\left(\eta_{H}, \lambda_{H}\right) d \lambda_{H} .
$$

By (30), for a walk in $n$ dimensions,

$$
\sum_{\lambda \in \text { chamber } \cap N \vec{z}+L} b_{\eta \lambda, N} /|S|^{N}
$$

is an approximate Riemann sum for the integral (32) with each term accurate to within $O\left(N^{(-m-n-2) / 2}\right)$, uniformly in $\lambda$. (The factor of $\operatorname{det} L$ in (30) disappears in taking the Riemann sum, because the Riemann sum is over a partition of the chamber into regions of volume $\operatorname{det} L$.) Similarly, by (31), for a walk restricted to $H$,

$$
\sum_{\lambda_{H} \in \text { chamber } \cap N \vec{z}+L} b_{\eta_{H} \lambda_{H}, N} /|S|^{N}
$$


is an approximate Riemann sum to the integral (37), with each term accurate to within $O\left(N^{(-m-n-1) / 2}\right)$. We cannot directly use the Riemann sums to evaluate the integrals, because the partition points $\lambda$ are evenly spaced rather than going to zero, and because the sums are infinite sums and the sum of the finite errors is not bounded.

For simplicity, we will work out the case (32) in full for the rest of this argument; the argument for (37) is analogous. We will show that the Riemann sum does give the correct limit, even when multiplied by the necessary constant $N^{m / 2}$; that is, we will show

$$
\lim _{N \rightarrow \infty}\left[\int_{\text {chamber }} N^{m / 2} b_{\kappa N}(\eta, \lambda) d \lambda-\sum_{\lambda \in \text { chamber } \cap N \vec{z}+L} \frac{N^{m / 2} b_{\eta \lambda, N}}{|S|^{N}}\right]=0 .
$$

The argument is similar to the proof of Lemma 2 in [6].

Choose any positive $\epsilon<1 / 2 n$, and split both the integral and the sum into the regions $|\lambda|<N^{1 / 2+\epsilon}$ and $|\lambda| \geq N^{1 / 2+\epsilon}$. For $|\lambda| \geq N^{1 / 2+\epsilon}$, we will show that both the sum and the integral in (40) go to zero; for $|\lambda|<N^{1 / 2+\epsilon}$, we will be able to use our Riemann sum to show that they converge to the same limit.

First look at the region $|\lambda| \geq N^{1 / 2+\epsilon}$. The integrand in (40) is $N^{m / 2}$ times the probability that a Brownian motion, started at $\eta$, will stay inside the chamber and be at $\lambda$ at time $\kappa N$. Ignoring the chamber, the integrand is certainly bounded by the probability that an unconstrained Brownian motion started at $\eta$ will be at $\lambda$ at time $\kappa N$. But we are integrating over the region $|\lambda| \geq N^{1 / 2+\epsilon}$, and the probability that Brownian motion gets outside that region goes to zero as $N$ goes to infinity faster than any polynomial in $N$ because of the $\exp \left(-|\lambda|^{2} /(2 \kappa N)\right)<\exp \left(-N^{2 \epsilon} /(2 \kappa)\right)$ term.

For the sum, we similarly bound the count of random walks. The summand is a constant multiple of $N^{m / 2}$ times the probability that a random walk, starting at $\eta$, will stay inside the Weyl chamber for $N$ steps, and will be at $\lambda$. This probability is clearly bounded by the probability that a random walk, starting at $\eta$, will be at $\lambda$ after $N$ steps. Summing that over $|\lambda| \geq N^{1 / 2+\epsilon}$ gives the probability that a random walk will be at radius greater than $N^{1 / 2+\epsilon}$ after $N$ steps. Again, any polynomial multiple of that probability goes to 0 as $N$ goes to infinity.

For the region $|\lambda|<N^{1 / 2+\epsilon}$, we make the change of variables $\vec{u}=\lambda / \sqrt{N}$, so that $\prod_{\alpha \in \Phi^{+}} \alpha(\lambda)=N^{m / 2} \prod_{\alpha \in \Phi^{+}} \alpha(\vec{u}), d \lambda=N^{n / 2} d \vec{u}$, and the region $|\lambda|<N^{1 / 2+\epsilon}$ is $|\vec{u}|<N^{\epsilon}$. Thus the integral in (40), which previously had a factor $N^{m / 2}$, becomes

$$
\int_{\text {chamber },|\vec{u}|<N^{\epsilon}} N^{(m+n) / 2} b_{\kappa N}(\eta, \sqrt{N} \vec{u}) d \vec{u} .
$$

Let $g(\vec{u})$ be the integrand. The limit (30) becomes

$$
\left|N^{(m+n) / 2} b_{\eta \sqrt{N} \vec{u}, N} /\left(\operatorname{det} L|S|^{N}\right)-g(\vec{u})\right|=O(1 / N) .
$$

Applying Lemma 1 to $g(\vec{u})$ after the change of variables, we have

$$
C \prod_{\alpha \in \Phi^{+}} \alpha(\vec{u}) \alpha(\eta) \exp \left(-\frac{|\vec{u}|^{2}}{2 \kappa}-\frac{|\eta|^{2}}{2 \kappa N}\right) / g(\vec{u})=1+O\left(N^{\epsilon-1 / 2}\right) .
$$


The only term in the numerator which depends on $N$ is $\exp \left(\left(-|\eta|^{2}\right) /(2 \kappa N)\right)$. This term is $1+O(1 / N)$, since $\eta$ is a constant and $\exp (c / N)$ is $1+O(1 / N)$. Thus we can get an approximate integrand which is independent of $N$,

$$
h(\vec{u})=C \prod_{\alpha \in \Phi^{+}} \alpha(\vec{u}) \alpha(\eta) \exp \left(\frac{-|\vec{u}|^{2}}{2 \kappa}\right),
$$

with the same degree of accuracy

$$
h(\vec{u}) / g(\vec{u})=1+O\left(N^{\epsilon-1 / 2}\right) .
$$

Since $g$ and $h$ are both positive on the entire chamber, the ratio of their integrals over the region $|\vec{u}|<N^{\epsilon}$ is also $1+O\left(N^{\epsilon-1 / 2}\right)$.

In addition, since $h$ decays exponentially, we have

$$
\int_{\text {chamber },|\vec{u}| \geq N^{\epsilon}} h(\vec{u}) d u=o\left(N^{c}\right) \text { for all } c,
$$

just as we had for $g$. Thus, in particular, we have

$$
\lim _{N \rightarrow \infty} \int_{\text {chamber }} g(\vec{u}) d u=\int_{\text {chamber }} h(\vec{u}) d u .
$$

The integral of $h$ is

$$
C \prod_{\alpha \in \Phi^{+}} \alpha(\eta) \int_{\text {chamber }} \prod_{\alpha \in \Phi^{+}} \alpha(\vec{u}) \exp \left(\frac{-|\vec{u}|^{2}}{2 \kappa}\right) d u,
$$

a constant multiple of $\prod_{\alpha \in \Phi^{+}} \alpha(\eta)$, and thus so is the limit of the integral of $g$. This proves Lemma 2.

We can now take a Riemann sum. Since $h$ is the product of a polynomial in $\vec{u}$ and a decreasing exponential, its gradient is bounded. The error in approximationg the integral of $h$ over a region of volume $V$ by a Riemann sum over partitions into subregions of diameter at most $\delta$ is at most $\sup (\nabla h) \delta V$, which is $O(\delta V)$. The volume of the region $|\vec{u}|<N^{\epsilon}$ is $O\left(N^{n \epsilon}\right)$, and the diameter of the partition in $(N \vec{z}+L) / \sqrt{N}$ is $O\left(N^{-1 / 2}\right)$. Thus we have

$$
\sum_{\vec{u} \in \text { chamber } \cap(N \vec{z}+L) / \sqrt{N},|\vec{u}|<N^{\epsilon}} \frac{\operatorname{det} L}{N^{n / 2}} h(\vec{u})-\int_{\text {chamber, },|\vec{u}|<N^{\epsilon}} h(\vec{u}) d \vec{u}=O\left(N^{n \epsilon-1 / 2}\right) .
$$

The approximation of $g$ for $h$ within this region is good to within a factor of $1+$ $O\left(N^{\epsilon-1 / 2}\right)$, and both are positive everywhere in the chamber, so the integrals of $g$ and $h$ agree to within the same factor, as do the sums of values of $g$ and $h$. Thus we have the same rate of convergence for the Riemann sum for $g$,

$$
\sum_{\vec{u} \in \text { chamber } \cap(N \vec{z}+L) / \sqrt{N},|\vec{u}|<N^{\epsilon}} \frac{\operatorname{det} L}{N^{n / 2}} g(\vec{u})-\int_{\text {chamber, }, \vec{u} \mid<N^{\epsilon}} g(\vec{u}) d \vec{u}=O\left(N^{n \epsilon-1 / 2}\right) .
$$


(It was necessary to go through $h$ because the factor of $N^{(m+n) / 2}$ prevents us from directly getting a uniform bound on the gradient of $g$ and thus a rate of convergence.)

And we are finally ready to use our approximate Riemann sum. For $|\vec{u}|<N^{\epsilon}$, we can take a sum over all $\vec{u}$ with $(N \vec{z}+L) / \sqrt{N}$ inside this region, using (42). We have at most $O\left(N^{\epsilon}\right)^{n} N^{n / 2}$ terms inside the region, since the lattice $L$ has dimension $n$. The approximate function value in (42) has an error of $O(1 / N)$, and this value is multiplied by the partition size det $L / N^{n / 2}$ for the Riemann sum. The sum of all errors is the product of these three factors, which is $O\left(N^{n \epsilon-1}\right)$. That is, we have

$$
\sum_{\substack{\vec{u} \in \operatorname{chamber} \cap(N \vec{z}+L) / \sqrt{N} \\|\vec{u}|<N^{\epsilon}}} \frac{\operatorname{det} L}{N^{n / 2}}\left[\frac{N^{(m+n) / 2} b_{\eta \sqrt{N} \vec{u}, N}}{\operatorname{det} L|S|^{N}}-g(\vec{u})\right]=O\left(N^{n \epsilon-1}\right) .
$$

Combining the Riemann sum from (50) with the approximation to the Riemann sum (51), and then undoing the change of variables, we have

$$
\lim _{N \rightarrow \infty}\left[\int_{\substack{\text { chamber } \\|\lambda|<N^{\epsilon+1 / 2}}} N^{m / 2} b_{\kappa N}(\eta, \lambda) d \lambda-\sum_{\substack{\lambda \in \text { chamber } \cap N \vec{z}+L \\|\lambda|<N^{\epsilon+1 / 2}}} \frac{N^{m / 2} b_{\eta \lambda, N}}{|S|^{N}}\right]=0 .
$$

This is the $|\lambda|<N^{\epsilon+1 / 2}$ part of (40), and since we have also shown that both sides go to zero for $|\lambda| \geq N^{\epsilon+1 / 2}$, we have proved (40).

Since we showed with the proof of Lemma 2 that the asymptotic for the integral is

$$
C N^{-m / 2} \prod_{\alpha \in \Phi^{+}} \alpha(\eta)
$$

the asymptotic for a random walk to stay within the Weyl chamber is also given by (53), with the same constant. This proves Theorem 1.

In the case of a walk restricted to the hyperplane $H$ for the Weyl group $A_{n-1}$, the calculations are almost the same. Throughout the calculations, we use $\lambda_{H}$ rather than $\lambda$, and the integral is over $n-1$ dimensions, so every term of $N^{-n / 2}$ becomes $N^{-(n-1) / 2}$ and we need $\epsilon<1 /(2(n-1))$. We split the integral in (37) into the same regions $\left|\lambda_{H}\right|<N^{1 / 2+\epsilon}$ and $\left|\lambda_{H}\right| \geq N^{1 / 2+\epsilon}$ as before; the sum and integral both go to zero on the region $\left|\lambda_{H}\right| \geq N^{1 / 2+\epsilon}$. The change of variables $\vec{u}_{H}=\vec{\lambda}_{H} / \sqrt{N}$ still gives $\prod_{\alpha \in \Phi^{+}} \alpha\left(\lambda_{H}\right)=N^{m / 2} \prod_{\alpha \in \Phi^{+}} \alpha\left(\vec{u}_{H}\right)$, but $d \lambda_{H}=N^{(n-1) / 2} d \vec{u}_{H}$. The error in (42) is still $O(1 / N)$. The error in the Riemann sums (49) and (50) is $O\left(N^{(n-1) \epsilon-1 / 2}\right)$. When we take the approximate Riemann sum, the partitions have volume $\operatorname{det} L / N^{(-n-1) / 2}$, and there are $O\left(N^{(-n+1) / 2+(n-1) \epsilon}\right)$ terms, so the error in the approximate sum is $O\left(N^{(n-1) \epsilon-1}\right)$. The limit (52) still holds, and thus so does Theorem 1.

\section{Asymptotics for Young tableaux}

Now that we have proved Theorem 1, the asymptotics for Young tableaux with at most $n$ rows follow immediately. Define $\eta=\mu+(-1,-2, \ldots,-n)$, so that a Young tableau of 
shape $\mu$ is a walk from $(-1,-2, \ldots,-n)$ to $\eta$. For a large $N$, Theorem 1 (with the $N$ in the theorem replaced by $N-k$ ) shows that the probability that a walk which is at $\eta$ will survive for $N-k$ more steps is asymptotic to

$$
C(N-k)^{-n(n-1) / 4} \prod_{1 \leq i<j \leq n}\left(\eta_{i}-\eta_{j}\right) .
$$

Equivalently, the number of tableaux of size $N$ with at most $n$ rows which contain a specific subtableau of shape $\mu$ is asymptotic to

$$
C n^{N-k}(N-k)^{-n(n-1) / 4} \prod_{1 \leq i<j \leq n}\left(\eta_{i}-\eta_{j}\right) .
$$

The constants and terms with $N$ are the same for all tableaux with $k$ entries, so the probability that a random tableau of size $N$ contains the subtableau $T$ of shape $\mu$ as its subtableau of size $k$ is proportional to the factor which depends on $\mu$,

$$
\prod_{1 \leq i<j \leq n}\left(\eta_{i}-\eta_{j}\right)=\prod_{1 \leq i<j \leq n}\left(\left(\mu_{i}-i\right)-\left(\mu_{j}-j\right)\right)
$$

proving (2).

For example, consider the simplest case, with just two rows. The two tableaux are $T={ }^{12}$ of shape $\mu=(2,0)$ and $T^{\prime}={ }_{2}^{1}$ of shape $\mu^{\prime}=(1,1)$. We thus have $\eta_{1}=1, \eta_{i}=-i$ for $i \geq 2$, and $\eta_{1}^{\prime}=0, \eta_{2}^{\prime}=-1, \eta_{i}^{\prime}=-i$ for $i \geq 3$.

The terms in the products in (56) for $\mu$ and $\mu^{\prime}$ are equal for $i \geq 3$, since $\mu_{i}=\mu_{i}^{\prime}$. We can thus compute the relative probabilities for $T$ and $T^{\prime}$ by computing the ratio of the terms for $i \leq 2$.

For $T$, we have

$$
\left(\eta_{1}-\eta_{2}\right) \prod_{j=3}^{n}\left(\eta_{1}-\eta_{j}\right)\left(\eta_{2}-\eta_{j}\right)=3 \prod_{j=3}^{n}(j+1)(j-2)=\frac{(n+1) !(n-2) !}{2} .
$$

For $T^{\prime}$, we have

$$
\left(\eta_{1}-\eta_{2}\right) \prod_{j=3}^{n}\left(\eta_{1}-\eta_{j}\right)\left(\eta_{2}-\eta_{j}\right)=1 \prod_{j=3}^{n} j(j-1)=\frac{n !(n-1) !}{2} .
$$

Thus we have

$$
\lim _{N \rightarrow \infty} \frac{P(n, N, T)}{P\left(n, N, T^{\prime}\right)}=\frac{(n+1) !(n-2) ! / 2}{n !(n-1) ! / 2}=\frac{n+1}{n-1} .
$$

Since $T$ and $T^{\prime}$ are the only two tableaux, the probabilities sum to 1 , so

$$
\lim _{N \rightarrow \infty} P(n, N, T)=\frac{n+1}{2 n} .
$$

Thus the probability that 2 is in the top row of a large random Young tableau with at most $n$ rows has limit $(n+1) / 2 n$. As the number of rows goes to infinity, this approaches the expected $1 / 2$. 
We will now do the general calculation to see what happens to the probability in (56) as the number $n$ of rows allowed becomes large compared to the number $r$ of rows in $\mu$. Let $\mu=\left(\mu_{1}, \ldots, \mu_{r}\right)$ (with $\mu_{i}=0$ allowed for some $i$ ), so that

$$
\eta=\left(\mu_{1}-1, \mu_{2}-2, \ldots, \mu_{r}-r,-(r+1),-(r+2), \ldots,-n\right) .
$$

We will now multiply the probability $\prod\left(\eta_{i}-\eta_{j}\right)$ by factors which depend only on $n$, $r$, and $k$; it will thus preserve the ratio of probabilities for any two tableaux with at most $r$ rows. When we know all of the probabilities up to a constant factor, we can find the constant because the sum of all probabilities must be 1 .

We insert these extra factors and define

$$
f_{n}(\mu)=\frac{1}{n^{k-r(r-1) / 2}(n !)^{r}} \prod_{1 \leq i<j \leq n}\left(\eta_{i}-\eta_{j}\right) \prod_{r+1 \leq i<j \leq n} \frac{1}{j-i} .
$$

To compute the limit $\lim _{n \rightarrow \infty} f_{n}(\mu)$, we will split the product $\prod\left(\eta_{i}-\eta_{j}\right)$ into three parts, according to whether $i>r, i \leq r<j$, or $j \leq r$.

If both $i$ and $j$ are greater than $r$, then $\left(\eta_{i}-\eta_{j}\right) /(j-i)=1$, so these factors cancel out in the product.

For a fixed $i \leq r$, the terms for $j>r$ are

$$
\prod_{j=r+1}^{n}\left(\eta_{i}-\eta_{j}\right)=\prod_{j=r+1}^{n}\left(\mu_{i}+j-i\right)=\frac{\left(\mu_{i}+n-i\right) !}{\left(\mu_{i}+r-i\right) !}
$$

We rewrite the product of all these terms in that form, and leave the $1 \leq i<j \leq r$ terms intact, to get

$$
f_{n}(\mu)=\frac{1}{n^{k-r(r-1) / 2}(n !)^{r}} \prod_{1 \leq i<j \leq r}\left(\left(\mu_{i}-i\right)-\left(\mu_{j}-j\right)\right) \prod_{i=1}^{r} \frac{\left(\mu_{i}+n-i\right) !}{\left(\mu_{i}+r-i\right) !} .
$$

The only terms which depend on $n$ in this product are

$$
\frac{1}{n^{k-r(r-1) / 2}} \prod_{i=1}^{r} \frac{\left(\mu_{i}+n-i\right) !}{n !}
$$

and since $\sum \mu_{i}=k$ and $\sum i=r(r-1) / 2$, we can write this as

$$
\prod_{i=1}^{r} \frac{\left(\mu_{i}+n-i\right) !}{n ! n^{\mu_{i}-i}}
$$

For each term $\left(\mu_{i}+n-i\right) ! / n ! n^{\mu_{i}-i}$, we cancel out as many factors as possible. If $\mu_{i}-i \geq 0$, then the entire $n$ ! in the denominator cancels out, leaving

$$
\frac{(n+1) \cdots\left(n+\mu_{i}-i\right)}{n^{\mu_{i}-i}} .
$$


The total number of factors in all the numerators is at most $\sum\left(\mu_{i}-i\right)=k$, and each factor $n+c$ can be paired with one $n$ in the denominator of the same term. Thus the product of all these terms is a product of at most $k$ terms $(n+c) / n$, which goes to 1 as $n$ goes to infinity.

Similarly, if $\mu_{i}-i<0$, then the factorial $\left(\mu_{i}+n-i\right)$ ! cancels out, leaving

$$
\frac{n^{i-\mu_{i}}}{\left(n+\mu_{i}-i+1\right) \cdots(n-1)} .
$$

The total number of factors in all the denominators is at most $\sum_{i=1}^{r} i=r(r-1) / 2$, and each factor $n-c$ can be paired with one $n$ in the numerator. Thus the product of all these terms is a product of at most $r(r-1) / 2$ terms $n /(n-c)$, which goes to 1 as $n$ goes to infinity. Thus the limit as $n$ goes to infinity of the product (65) also goes to 1 .

We thus have

$$
\lim _{n \rightarrow \infty} f_{n}(\mu)=\prod_{1 \leq i<j \leq r}\left(\left(\mu_{i}-i\right)-\left(\mu_{j}-j\right)\right) \prod_{i=1}^{r} \frac{1}{\left(\mu_{i}+r-i\right) !} .
$$

And this value is exactly $f^{\mu} / k ![4,(4.11)]$. Since the computation is valid even if some $\mu_{i}=0$, we get the same limits by pretending that all tableaux with $k$ cells have $k$ rows, some of length zero.

Thus the probability that a given tableau of shape $\mu$ is a subtableau of a randomly chosen large tableau with at most $n$ rows approaches $f^{\mu} / k$ ! as $n$ goes to infinity.

Note that this confirms, but does not quite prove, the result of [12]. If we let $P(n, N, T)$ be the probability that a randomly chosen tableau with $N$ cells and at most $n$ rows contains $T$ as a subtableau, then we have proved

$$
\lim _{n \rightarrow \infty} \lim _{N \rightarrow \infty} P(n, N, T)=\frac{f^{\mu}}{k !} .
$$

The result of [12] would have the limits in the opposite order. If $N \leq n$, the restriction to $n$ rows does not matter, and thus $\lim _{n \rightarrow \infty} P(n, N, T)=P(N, T)$.

\section{$5 \quad$ Asymptotics for up-down tableaux}

We have an analogous result for the other classical Weyl chambers. For $B_{n}$, we let $\eta=\mu+(n, n-1, \ldots, 1)$, so that an up-down tableau of final shape $\mu$ corresponds to a walk from $(n, n-1, \ldots, 1)$ to $\eta$ with steps $\pm e_{i}$ in the chamber $x_{1}>x_{2}>\cdots>x_{n}>0$. Thus the probability that a random up-down tableau of size $N$ with at most $n$ rows at all steps will have a particular subtableau $\mu$ at step $k$ is proportional to

$$
\prod_{1 \leq i<j \leq n}\left(\eta_{i}-\eta_{j}\right)\left(\eta_{i}+\eta_{j}\right) \prod_{i=1}^{n} \eta_{i}
$$


We can use the same argument as above to take the limit of these probabilities as $n \rightarrow \infty$. Since the size of the partition $\mu$ no longer determines the number of steps $k$ to reach it, we introduce a new variable $k^{\prime}=\sum \mu_{i}$.

Note that $k^{\prime}$ depends on the specific tableau chosen, and thus when we compute a term of $(2 n)^{k^{\prime}}$ in the asymptotic probability, this will show a dependency on $k^{\prime}$. Since $k^{\prime} \leq k$, with equality only if our tableau is standard (with upward steps only), this will show that the probability that the tableau at step $k$ is standard goes to 1 as the number of rows goes to infinity.

We gather all the necessary factors into one expression. As we did with standard tableaux, we will multiply by extra factors which depend only on $n$ and $r$ to get an expression $f_{n}^{\prime}(\mu)$ which depends only on $n$ and $r$ and preserves the ratio of probabilities for any two up-down tableaux with at most $r$ rows. However, $k^{\prime}$ depends on $\mu$, not just on $n$ and $r$, and thus we cannot multiply by a factor which depends on $k^{\prime}$; this accounts for the dependence of the limit on $k^{\prime}$. We define

$$
f_{n}^{\prime}(\mu)=\frac{1}{n^{-r(r-1) / 2}(n !)^{r+1}} \prod_{r+1 \leq i<j \leq n} \frac{1}{j-i} \prod_{1 \leq i<j \leq n}\left(\eta_{i}-\eta_{j}\right) \frac{\eta_{i}+\eta_{j}}{2 n+2-i-j} \prod_{i=1}^{n} \eta_{i} .
$$

The $\left(\eta_{i}-\eta_{j}\right)$ factors are the same factors as for standard tableaux, since $\eta_{i}=\mu_{i}+$ $(n+1)-i$ and it was $\mu_{i}-i$ for standard tableaux. That is, (69) gives

$$
\begin{aligned}
\lim _{n \rightarrow \infty} \frac{1}{n^{k^{\prime}-r(r-1) / 2}(n !)^{r}} \prod_{1 \leq i<j \leq n}\left(\eta_{i}-\eta_{j}\right) & \prod_{r+1 \leq i<j \leq n} \frac{1}{j-i} \\
& =\prod_{1 \leq i<j \leq r}\left(\left(\mu_{i}-i\right)-\left(\mu_{j}-j\right)\right) \prod_{i=1}^{r} \frac{1}{\left(\mu_{i}+r-i\right) !} .
\end{aligned}
$$

Note the $n^{k^{\prime}}$ in the denominator; we could not introduce this factor into $f_{n}^{\prime}(\mu)$, and this is why the final asymptotic will have a factor of $n^{k^{\prime}}$.

For the $\eta_{i}$ terms, we use the remaining factor of $1 / n$ ! from $f_{n}^{\prime}(\mu)$. We have

$$
\frac{1}{n !} \prod_{i=1}^{n}\left(\eta_{i}\right)=\prod_{i=1}^{n} \frac{\eta_{i}}{n+1-i}=\prod_{i=1}^{r} \frac{n+1-i+\mu_{i}}{n+1-i}
$$

and this has limit 1 as $n$ goes to infinity.

For the $\eta_{i}+\eta_{j}$ terms, we use the extra factors of $2 n+2-i-j$ in the denominator of $f_{n}^{\prime}(\mu)$; the product of these factors over all $i$ and $j$ again depends only on $n$. We now look separately at the terms $\left(\eta_{i}+\eta_{j}\right) /(2 n+2-i-j)$ for $i>r, i \leq r<j$, and $j \leq r$. If $r<i<j$, then $\left(\eta_{i}+\eta_{j}\right) /(2 n+2-i-j)=1$. For the terms with $i$ fixed and at most $r$ 
but $j>r$, we have

$$
\begin{aligned}
\prod_{j=r+1}^{n} \frac{\eta_{i}+\eta_{j}}{2 n+2-i-j} & =\prod_{j=r+1}^{n} \frac{\mu_{i}+2 n+2-i-j}{2 n+2-i-j} \\
& =\frac{\left(\mu_{i}+2 n+1-i-r\right) ! /\left(\mu_{i}+n+1-i\right) !}{(2 n+1-i-r) ! /(n+1-i) !} \\
& =\frac{\left(\mu_{i}+2 n+1-i-r\right) !}{(2 n+1-i-r) !} \frac{(n+1-i) !}{\left(\mu_{i}+n+1-i\right) !} .
\end{aligned}
$$

After cancelling terms, the first fraction in the final expression is a product of $\mu_{i}$ terms between $2 n+2-i-r$ and $\mu_{i}+2 n+1-i-r$, and the second is the reciprocal of a product of $\mu_{i}$ terms between $n+2-i$ and $\mu_{i}+n+1-i$. Thus we can pair these off to get a product of $\mu_{i}$ terms of the form $\left(2 n+c_{1}\right) /\left(n+c_{2}\right)$, and as $n$ goes to infinity, each term approaches 2 . There are a total of $\sum \mu_{i}=k^{\prime}$ such terms, independent of $n$ or $r$, so as $n$ goes to infinity, the product approaches $2^{k^{\prime}}$.

For the terms with $i<j \leq r$, we have

$$
\prod_{1 \leq i<j \leq r} \frac{\eta_{i}+\eta_{j}}{2 n+2-i-j}=\prod_{1 \leq i<j \leq r} \frac{\mu_{i}+\mu_{j}+2 n+2-i-j}{2 n+2-i-j} .
$$

There are only $r(r-1) / 2$ of these terms, and each one approaches 1 as $n$ goes to infinity, so the product approaches 1 . Putting the three factors together, we have

$$
\lim _{n \rightarrow \infty} \prod_{1 \leq i<j \leq n} \frac{\eta_{i}+\eta_{j}}{2 n+2-i-j}=2^{k^{\prime}}
$$

As we multiply these terms together, we have picked up factors of $n^{k^{\prime}}$ from the $\eta_{i}-\eta_{j}$ terms, and $2^{k^{\prime}}$ from the $\eta_{i}+\eta_{j}$ terms, in addition to the product terms involving $\mu_{i}$. Thus for this case, we have

$$
\lim _{n \rightarrow \infty} \frac{f_{n}^{\prime}(\mu)}{(2 n)^{k^{\prime}}}=\prod_{1 \leq i<j \leq r}\left(\left(\mu_{i}-i\right)-\left(\mu_{j}-j\right)\right) \prod_{i=1}^{r} \frac{1}{\left(\mu_{i}+r-i\right) !} .
$$

As for the standard tableaux, this limit is independent of our choice of $r$. However, the limit is not directly proportional to the limiting probability that a random up-down tableau contains $T$ of shape $\mu$ as a subtableau at step $k$, because of the extra $(2 n)^{k^{\prime}}$ term.

In particular, if we have tableaux $T$ and $\hat{T}$ of shapes $\mu$ and $\hat{\mu}$ with different numbers of cells, so that $k^{\prime}>\hat{k}^{\prime}$, then the different powers of $n$ in (78) show that

$$
\lim _{n \rightarrow \infty} \frac{f_{n}^{\prime}(\mu)}{f_{n}^{\prime}(\hat{\mu})}=\infty
$$

Therefore, the probability goes to 1 that we will have a tableau with the maximum number of cells $k^{\prime}=k$. 
If $k^{\prime}=k$, we have

$$
\lim _{n \rightarrow \infty} \lim _{N \rightarrow \infty} P(n, N, T)=C_{k} \prod_{1 \leq i<j \leq r}\left(\left(\mu_{i}-i\right)-\left(\mu_{j}-j\right)\right) \prod_{i=1}^{r} \frac{1}{\left(\mu_{i}+r-i\right) !}
$$

with a proportionality constant $C_{k}$ such that the sum over all tableaux of size $k$ is 1 . And this is the same limit as in (69), so we do have

$$
\lim _{n \rightarrow \infty} \lim _{N \rightarrow \infty} P(n, N, T)=\frac{f^{\mu}}{k !}
$$

for up-down tableaux as well, provided that $T$ has $k$ cells. We conjecture that this is the limiting probability for up-down tableaux with an unlimited number of rows, as it is for standard tableaux.

Let $P_{k^{\prime}}(n, N, T)$ be the probability that $T$ is the subtableau at step $k$, given that the subtableau at step $k$ has exactly $k^{\prime}$ cells. For any fixed $n$, we are conditioning on an event

of nonzero probability, so this is meaningful, and the limit in (78), with the $(2 n)^{k^{\prime}}$ term now discarded, gives

$$
\lim _{n \rightarrow \infty} \lim _{N \rightarrow \infty} P_{k^{\prime}}(n, N, T)=C_{k^{\prime}} \prod_{1 \leq i<j \leq r}\left(\left(\mu_{i}-i\right)-\left(\mu_{j}-j\right)\right) \prod_{i=1}^{r} \frac{1}{\left(\mu_{i}+r-i\right) !},
$$

with a proportionality constant $C_{k^{\prime}}$ such that the sum over all tableaux of size $k^{\prime}$ is 1 . Again, we have

$$
\lim _{n \rightarrow \infty} \lim _{N \rightarrow \infty} P_{k^{\prime}}(n, N, T)=\frac{f^{\mu}}{k^{\prime} !}
$$

This limit is proportional to the number of standard tableaux of shape $\mu$. In fact, it is also proportional to the number of up-down tableaux of shape $\mu$ among all tableaux with size $k$ and $k^{\prime}$ cells, because that value is [15]

$$
\tilde{f}_{k}^{\mu}=f^{\mu}\left(\begin{array}{c}
k \\
k^{\prime}
\end{array}\right) \prod_{t=1}^{\frac{k-k^{\prime}}{2}-1}(2 t-1)
$$

and every factor except $f^{\mu}$ in this expression depends only on $k$ and $k^{\prime}$.

For $D_{n}$, we let $\eta=\mu+(n-1, n-2, \ldots, 0)$. The $D_{n}$ equivalent of an up-down tableau is an up-down sequence of Young-like diagrams in which the $n$th row may have a negative number of boxes as long as the combined number of boxes in the last two rows is nonzero. I am not aware of the tableaux being defined as such, although they do again give the multiplicity of the representation with highest weight $\mu$ in the $k$ th tensor power of the defining representation of $S_{2 n}$ [7]. The probability that a walk which survives for $N$ steps passes through $\eta$ after $k$ steps is proportional to

$$
\prod_{1 \leq i<j \leq n}\left(\eta_{i}-\eta_{j}\right)\left(\eta_{i}+\eta_{j}\right)
$$


The limit as $n$ goes to infinity is less interesting in this case, because the allowed tableaux are different for each $n$. The problem with an unlimited number of rows is not meaningful, as the last row can never be used if the number of rows is unlimited, and thus the $D_{n}$ problem reduces to the $B_{n}$ problem. By essentially the same argument as for $B_{n}$ (using factors of $2 n-i-j$ rather than $2 n+2-i-j$ in the factors in $f_{n}^{\prime}(\mu)$ ), we can show that the limiting probability for $D_{n}$ as $n$ goes to infinity is the same limit (81) as for $B_{n}$, and if we restrict to tableaux with $k^{\prime}$ cells, we get the limit (83).

Acknowledgments. I would like to thank Christian Krattenthaler and the referee for several useful suggestions.

\section{References}

[1] Berele, A., "A Schensted-type correspondence for the symmetric group," J. Combin. Th. A 43(1986), 320-328.

[2] Biane, P., "Minuscule weights and random walks on lattices," Quant. Prob. Rel. Topics 7(1992) 51-65.

[3] N. Bourbaki, Groupes et Algebres de Lie, Chapters 4,5,6, Hermann, Paris, 1968.

[4] Fulton, W., and Harris, J., Representation Theory: A First Course, Springer-Verlag, New-York-Heidelberg-Berlin, 1991.

[5] Gessel, I. M., and Zeilberger, D., "Random walk in a Weyl chamber," Proc. Amer. Math. Soc. 115(1992), 27-31.

[6] Grabiner, D. J., "Brownian motion in a Weyl chamber, non-colliding particles, and random matrices," Annales de l'I.H.P., Prob. et Stat. 35(1999), 177-204.

[7] Grabiner, D. J., and Magyar, P, "Random walks in Weyl chambers and the decomposition of tensor powers," J. Alg. Combin. 2(1993) 239-260.

[8] Humphreys, J. E., Reflection groups and Coxeter groups, Cambridge University Press, 1990.

[9] Jaggard, A. D., "Subsequence containment by involutions," Electron. J. Combin. 12(1)(2005), \#R14.

[10] Kuperberg, G., "Random words, quantum statistics, central limits, random matrices," Methods Appl. Anal., 9(2002), 101-119.

[11] Lawler, G.F., Intersections of random walks, Birkhäuser, Boston-Basel-Stuttgart, 1991.

[12] McKay, B. D., Morse, J., and Wilf, H. S., "The distributions of the entries of Young tableaux,' J. Combin. Th. A, 97(2002), 117-128.

[13] Proctor, R. A., "Reflection and algorithm proofs of some more Lie group dual pair identities," J. Combin. Th. A 62(1993), 107-127.

[14] Stanley, R. P., "On the enumeration of skew Young tableaux," Adv. Appl. Math. 30(2003), 283-294.

[15] Sundaram, S. "The Cauchy identity for $S p(2 n)$, , J. Combin. Th. A 53(1990), 209238. 